\title{
Correction \\ Correction: Kang et al. Preparation and In Vivo Evaluation of a Lidocaine Self-Nanoemulsifying Ointment with Glycerol Monostearate for Local Delivery. Pharmaceutics 2021, 13, 1468
}

Ji-Hyun Kang ${ }^{1}{ }^{(D}$, Kwang-Hwi Yoo ${ }^{1}$, Hyo-Young Park ${ }^{2}$, Seung-Min Hyun ${ }^{2}$, Sang-Duk Han ${ }^{2}$, Dong-Wook Kim ${ }^{3, *}$ and Chun-Woong Park $1, *$ (D)

check for

updates

Citation: Kang, J.-H.; Yoo, K.-H.;

Park, H.-Y.; Hyun, S.-M.; Han, S.-D.

Kim, D.-W.; Park, C.-W. Correction:

Kang et al. Preparation and In Vivo

Evaluation of a Lidocaine

Self-Nanoemulsifying Ointment with

Glycerol Monostearate for Local

Delivery. Pharmaceutics 2021, 13, 1468

Pharmaceutics 2022, 14, 155.

https://doi.org/10.3390/

pharmaceutics 14010155

Received: 26 November 2021

Accepted: 5 January 2022

Published: 10 January 2022

Publisher's Note: MDPI stays neutral with regard to jurisdictional claims in published maps and institutional affiliations.

Copyright: (c) 2022 by the authors. Licensee MDPI, Basel, Switzerland. This article is an open access article distributed under the terms and conditions of the Creative Commons Attribution (CC BY) license (https:// creativecommons.org/licenses/by/ $4.0 /)$.
1 College of Pharmacy, Chungbuk National University, Cheongju 28160, Korea; jhkanga@naver.com (J.-H.K.); dbrhkdgnl1@naver.com (K.-H.Y.)

2 DONG-A Pharm. Co., Ltd., Yongin 17073, Korea; Hyogoo312@donga.co.kr (H.-Y.P.); hsm@donga.co.kr (S.-M.H.); tilldie@donga.co.kr (S.-D.H.)

3 Department of Pharmaceutical Engineering, Cheongju University, Cheongju 28503, Korea

* Correspondence: pharmengin@cju.ac.kr (D.-W.K.); cwpark@cbnu.ac.kr (C.-W.P.); Tel.: +82-43-229-7984 (D.-W.K.); +82-43-261-3330 (C.-W.P.);

Fax: +82-43-229-8577 (D.-W.K.); +82-43-268-2732 (C.-W.P.)

The authors wish to make the following corrections to this paper [1].

In the original publication, there was a mistake in the legend for Table 1 as published, dl-Methylephedrine $\mathrm{HCl}$ of L4 is " 0.5 " and Petrolatum of L4 is "91.45". The corrected Table 1 appears below

Table 1. Formulation and rheological characterization of lidocaine ointments.

\begin{tabular}{ccccccc}
\hline Excipient. & L1 & L2 & L3 & L4 & L5 & L6 \\
\hline Petrolatum & 95.00 & 91.00 & 86.00 & 91.95 & 86.45 & 81.45 \\
MCT oil & 1.00 & 1.00 & 1.00 & 1.00 & 1.00 & 1.00 \\
Glycerol monostearate & - & 5.00 & 10.00 & - & 5.00 & 10.00 \\
Vitamin E-acetate & - & - & - & 3.00 & 3.00 & 3.00 \\
Lidocaine base & 3.00 & 3.00 & 3.00 & 3.00 & 3.00 & 3.00 \\
Allantoin & - & - & - & 1.00 & 1.00 & 1.00 \\
Prednisolone acetate & - & - & - & 0.05 & 0.05 & 0.05 \\
dl-Methylephedrine HCl & - & - & - & - & 0.5 & 0.5 \\
pH & 8.4 & 8.4 & 8.5 & 8.5 & 8.5 & 8.5 \\
Viscosity $\left(\times 10^{3}\right.$ cP) & - & - & - & $23.5 \pm 0.5$ & $41.9 \pm 0.8$ & $95.2 \pm 0.9$ \\
Minimum extrusion & - & - & - & $19.5 \pm 0.2$ & $25.2 \pm 0.8$ & $54.9 \pm 0.9$ \\
force $(\mathrm{N})$ & & & & & &
\end{tabular}

Abbreviations: MCT, medium-chain triglyceride, $\mathrm{HCl}$, hydrochloride.

There was an error in the original article in Figure 1, "Lidcocaine" is misspelled as a legend. The correct Figure 1 appears below. 


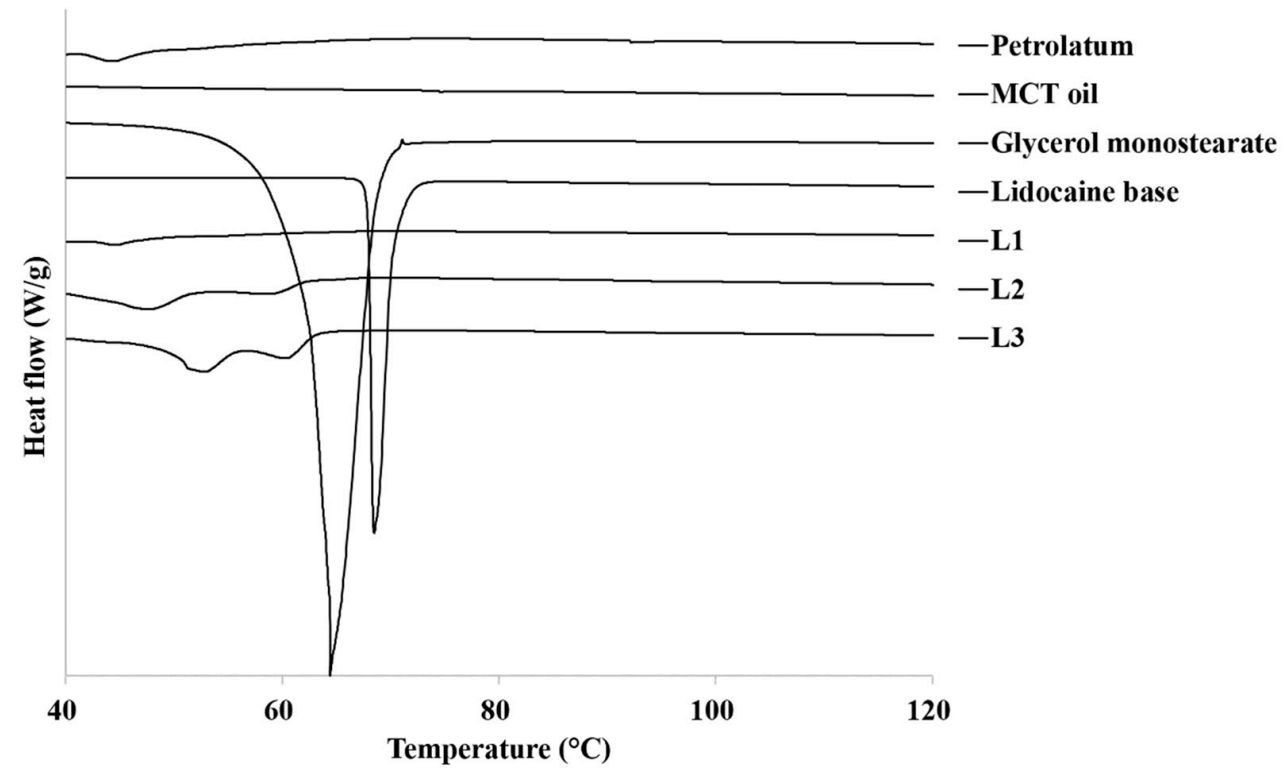

Figure 1. DSC thermograms of raw materials and prepared lidocaine ointments. MCT, mediumchain triglyceride.

There was an error in the original article in Figure 2, "Lidcocaine" is misspelled as a legend. The correct Figure 2 appears below.

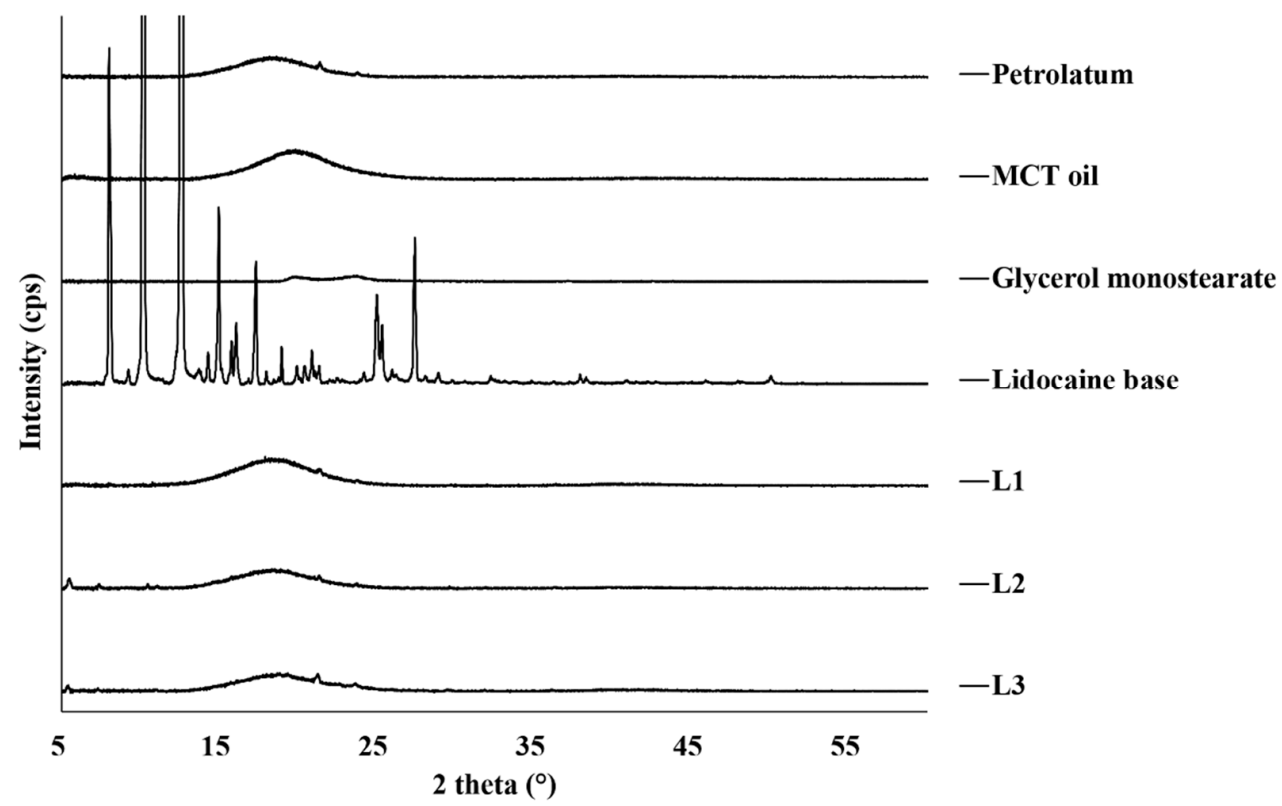

Figure 2. X-ray diffraction patterns of raw materials and prepared lidocaine ointments. MCT, medium-chain triglyceride.

The authors apologize for any inconvenience caused and state that the scientific conclusions are unaffected. The original publication has also been updated.

\section{Reference}

1. Kang, J.-H.; Yoo, K.-H.; Park, H.-Y.; Hyun, S.-M.; Han, S.-D.; Kim, D.-W.; Park, C.-W. Preparation and in vivo evaluation of a lidocaine self-nanoemulsifying ointment with glycerol monostearate for local delivery. Pharmaceutics 2021, 13, 1468. [CrossRef] [PubMed] 